\title{
Sleep in Children with Neurodevelopmental Disabilities
}

\author{
Marco Angriman ${ }^{1}$ Barbara Caravale ${ }^{2}$ Luana Novelli ${ }^{2}$ Raffaele Ferri ${ }^{3}$ Oliviero Bruni ${ }^{2}$
}

\footnotetext{
${ }^{1}$ Department of Pediatrics, Child Neurology and Neurorehabilitation Unit, Central Hospital of Bolzano, Italy

2 Department of Developmental and Social Psychology, Sapienza University, Rome, Italy

${ }^{3}$ Department of Neurology, I.C., Oasi Institute (IRCCS), Sleep Research Centre, Troina, Italy
}

Neuropediatrics 2015;46:199-210.
Address for correspondence Oliviero Bruni, MD, Department of Developmental and Social Psychology, Sapienza University, Via dei Marsi 78, 00185 Rome, Italy (e-mail: oliviero.bruni@uniroma1.it).

\author{
Abstract \\ Keywords \\ - sleep disorders \\ - neurodevelopmental \\ disabilities \\ - Down syndrome \\ - Fragile X syndrome \\ - Prader-Willi \\ syndrome \\ - Angelman syndrome \\ - Rett syndrome \\ - Smith-Magenis \\ syndrome \\ - cerebral palsy \\ - autism spectrum \\ disorders
}

This review describes recent research in pediatric sleep disorders associated with neurodevelopmental disabilities (NDDs) and their treatment. NDDs affect more than $2 \%$ of the general population and represent more than $35 \%$ of the total cases of children referred to a neuropsychiatric center for sleep problems. Specific clinical and therapeutic aspects of sleep disorders associated with Down syndrome, Fragile $X$ syndrome, Prader-Willi syndrome, Angelman syndrome, Rett syndrome, Smith-Magenis syndrome, cerebral palsy, and autism spectrum disorders are described. Furthermore, the drugs commonly used for sleep disorders in children with NDDs are described. The review clearly highlighted that children with NDDs are often affected by sleep disorders that require appropriate clinical and therapeutic approach to improve quality of life in both patients and families.

\section{Introduction}

Sleep disorders in children with neurodevelopmental disabilities (NDDs) have raised increasing interest in recent years, especially some specific conditions such as Down syndrome (DS), Fragile-X syndrome, Rett syndrome (RS), Prader-Willi syndrome (PWS), Angelman syndrome (AS), tuberous sclerosis, and autism spectrum disorders (ASDs).

NDDs affect approximately $2 \%$ of the general population and are associated with varying degrees of cognitive, physical, and emotional impairment. ${ }^{1}$

Sleep disturbances in children with NDDs are highly prevalent and are linked to a multifactorial etiology with different contributions of neurologic, medical, and psychiatric comorbidities. Sleep difficulties can result in additional learning and behavior problems, affecting the whole family's health and well-being. ${ }^{2}$

Furthermore, differently from age-related sleep disturbances in typically developing children, sleep disorders in patients with NDDs tend to be chronic, lasting into adolescence or adulthood.

Despite these conditions associated with different developmental disorders constitute more than $35 \%$ of total cases of children referred to a neuropsychiatric center for sleep problems, research on the treatment of sleep disorders in children with NDDs has been relatively scarce. ${ }^{3}$ received

December 24, 2014 accepted after revision January 31, 2015 published online April 28, 2015
Issue Theme Sleep and

Neurodevelopmental Disorders; Guest Editor, Oskar G. Jenni, MD (c) 2015 Georg Thieme Verlag KG
Stuttgart · New York

DOI http://dx.doi.org/ 10.1055/s-0035-1550151. ISSN $0174-304 X$. 
The pathophysiology of sleep disorders in children with NDDs might be linked to the specific syndrome genotype or phenotype, to an endogenous dysfunction in hormone and neurotransmitter release or to altered perception of the zeitgebers (light-dark cycle, food schedule, maternal inputs, etc.).

In some patients with NDDs, problematic sleep is a phenotypic characteristic of a particular disorder or genetic condition and the knowledge of the distinctive features of sleep disorders in patients with NDDs is crucial for their effective treatment. ${ }^{4}$

The main sleep complaints in children with NDDs are represented by difficulty in settling at night (51\%) and nocturnal awakenings (67\%). ${ }^{5}$ Fragmented sleep throughout the day and night determines daytime sleepiness and irregular sleep schedule that may lead to a free-running rhythm or to a complete reversal of the night-day cycle. ${ }^{6}$

\section{Sleep Abnormalities in Children with Genetic Disorders}

\section{Down Syndrome}

Several studies have shown that children with DS are affected by different sleep disturbances. The most common of them is represented by obstructive sleep apnea syndrome (OSAS) that affects approximately 50 to $80 \%$ of people with DS. ${ }^{7,8}$ This high prevalence is linked to the definite physical characteristics of DS, such as, craniofacial and upper airway abnormalities, obesity, generalized hypotonia, tonsil and adenoid hypertrophy, macroglossia, glossoptosis, hypoplastic trachea, midfacial, and mandibular hypoplasia. ${ }^{9-11}$

In addition, Ferri et al hypothesized a brain stem dysfunction in DS, responsible for the abnormal imbalance between the sympathetic and vagal systems and confirms the presence of central as opposed to obstructive apnea in subjects with DS without chronic respiratory pathology. ${ }^{12}$ These central events were preceded by sighs, but they provoked significant oxygen desaturation.

Excessively fragmented sleep, problems with sleep maintenance, early waking, daytime sleepiness, and decreased leg motor activity have also been described in infants with DS versus typically developing infants. ${ }^{13-16}$

Sleep problems have been often described as correlated with other problems such as asthma, autism, and a history of enlarged adenoids and tonsils.

A recent study showed that although a questionnaire survey reported that $65 \%$ of the children had significant sleep problems in the preceding month, the parents often did not report sleeping difficulties in their children. About $46 \%$ of children with DS screened positive for sleep-related breathing problems and $21 \%$ for sleep-related movement disorders. Sleep disorders represent an underrecognized problem in children with DS and the knowledge of comorbidities may help to deal with sleep problems in this population. ${ }^{9}$

Polysomnographic (PSG) studies showed that children with DS had significantly less rapid eye movement (REM) sleep than typically developing children and presented a low $\mathrm{R}$ index (number of high-frequency REMs against number of low-frequency REMs). ${ }^{17}$ In addition, they showed an increase in wake after sleep onset, body movements, and fewer spindle bursts. ${ }^{18}$

Recent practice parameters of the American Academy of Pediatrics recommend to discuss with parents, at least once during the first 6 months of life, symptoms of obstructive sleep apnea (OSA), including heavy breathing, snoring, uncommon sleep positions, frequent night awakening, daytime sleepiness, apneic pauses, and behavior problems that could be associated with poor sleep. Moreover, screening for signs of OSA should be included in every cardiologic follow-up. ${ }^{19}$

\section{Angelman Syndrome}

AS is a neurodevelopmental genetic disorder caused by the absence or loss of function of the maternally inherited allele at the 15q11-q13 domain; it occurs in $1: 12,000$ to 20,000 individuals and accounts for $6 \%$ of all children with severe cognitive disability and epilepsy. ${ }^{20,21}$

AS is characterized by developmental delay, severe speech impairment, profound intellectual disability, ataxic gait, and/ or tremulous movements of limbs, a peculiar behavior with frequent laughter, apparently happy temperament, hand flapping and jerky movements (puppet-like movements), and severe epilepsy. ${ }^{22}$ Other clinical features are widely spaced teeth, an open mouth, tongue protrusion, microcephaly, fair skin and hair, and hyperactivity.

Sleep disturbances are common in AS and are included in the diagnostic criteria established in 1995 and updated in $2005.23,24$

The few studies available on sleep disorders in AS show a high frequency of disorders of initiating and maintaining sleep, prolonged sleep latency, prolonged wakefulness after sleep onset, high number of night awakenings, and reduced total sleep time. ${ }^{25}$ In addition, snoring and parasomnias were frequently reported, including enuresis, bruxism, sleep terrors, somnambulism, and nocturnal hyperkinesia. ${ }^{26}$ Sleep problems appear more relevant and severe during childhood but in some patients they may persist into adulthood. ${ }^{27,28}$

Frequent sleep complaints include the following: difficulty initiating or maintaining sleep; frequent nighttime awakenings; irregular sleep/wake cycles, and circadian rhythm disorders, reduced sleep duration and nocturnal behaviors, including nocturnal laughing, sleepwalking/sleep terrors, bruxism, and seizures. ${ }^{24,26,27,29-31}$

PSG studies showed a significant reduction in sleep efficiency and in percentage and duration of REM sleep, whereas the percentage of SWS was found to be significantly higher, because of the presence of the 1 to $3 \mathrm{~Hz}$ bursts that represent the typical electroencephalography (EEG) pattern of the syndrome ${ }^{25}$; no respiratory abnormalities were found, but a tendency for subjects with AS to present a higher periodic limb movement index versus the other two control groups with epilepsy and mental retardation (MR) was found. ${ }^{32}$

In children with AS, biological alterations are causes of insomnia; treatment with sleep hygiene, behavioral therapy, and reinforcing of the sleep wake rhythm was documented to be as effective as hypnotic drugs. ${ }^{33,34}$ Furthermore, response to melatonin treatment in small trials suggests the possibility 
of altered melatonin secretion. ${ }^{35}$ In a recent study correlating sleep-wake pattern with serum melatonin levels, children with AS reported circadian rhythm sleep disorders (CRSDs), delayed sleep phase syndrome (DSPS), and free-running rhythm; the nighttime serum melatonin levels of patients with AS were significantly low and patients with AS with DSPS showed delayed melatonin peak. ${ }^{31}$

\section{Prader-Willi Syndrome}

PWS is a genetic disorder affecting in $1 / 10,000$ and $1 / 25,000$ live births. ${ }^{36,37}$ The genetic defect is linked to a deletion in the paternally inherited chromosome 15q11-q13 in 70 to $75 \%$ of individuals, to a maternal uniparental disomy in 20 to $25 \%$ of cases, and to abnormal methylation of the imprinting center on chromosome 15 in 1 to $2 \%$.

The early symptoms are represented by feeding difficulties and hypotonia followed by developmental delay, hypogonadism, hyperphagia, obesity and behavioral, and learning and psychological problems during childhood and adolescence. ${ }^{38}$

Different studies have been performed to evaluate sleep in patients with PWS showing a decrease of sleep and REM latency and the presence of sleep-onset REM periods, corroborating the hypothesis of a primary disorder of vigilance. ${ }^{39}$

A recent study that evaluated sleep microstructure in children with PWS by means of Cyclic Alternating Pattern (CAP) analysis showed a decrease of nonrapid eye movement (NREM) sleep instability, further supporting the hypothesis of the presence of a primary disorder of vigilance and underlying the similarities with narcolepsy. In these patients, growth hormone $(\mathrm{GH})$ therapy was able to increase the NREM sleep instability with the normalization in slow wave sleep. ${ }^{40}$

Obesity and hypothalamic dysfunction could be responsible for the primary abnormalities of ventilation during sleep that might contribute to excessive daytime sleepiness (EDS). Although EDS has similarity with narcolepsy, patients with PWS do not present the other typical symptoms of narcolepsy. ${ }^{39}$

Sleep-disordered breathing and a decreased response to hypoxia or hypercapnia has been described in patients with PWS. ${ }^{41}$ The studies on the prevalence of OSAS in PWS showed conflicting results: some reported a prevalence of approximately $90 \%$ whereas other studies reported a lower prevalence. $^{39}$

It seems that OSAS is a feature that appears during the development; in fact, infants with PWS, when compared with older children, were more likely to experience central sleep apnea other than obstructive events. ${ }^{42}$

With age, obesity increases and OSAS emerges-a recent review, analyzing 14 studies in children with PWS reported an OSAS prevalence of $79.91 \%-53.07 \%$ had mild OSAS, $22.35 \%$ moderate OSAS, and $24.58 \%$ severe OSAS. Narcolepsy was found to occur in $35.71 \%$ of children with PWS. Adenotonsillectomy was found to be effective in reducing OSAS for some children, but residual OSAS was present in the majority of cases after the surgery. ${ }^{43}$

Several studies reported that GH therapy in children with PWS might determine hypertrophy of tonsils or adenoids, increase OSA, and could be responsible for sudden death of subjects with PWS..$^{39,44,45}$ In a recent study, it was reported that, although the percentage of patients with an obstructive apnea-hypopnea index $>1$ increased during GH treatment, a decrease of respiratory disturbance index and the central apnea index were observed; the authors concluded that longterm GH treatment in patients with PWS is generally safe, recommending annual polysomnography and adenotonsillar evaluation. $^{45}$

Because of the concurrent presence of different sleep disturbances (EDS, narcolepsy, and sleep-disordered breathing) in children with PWS, the approach should be multidisciplinary. GH treatment is now considered to be safe, but it is still contraindicated in patients with PWS who are severely obese or have a severe respiratory impairment. EDS can be successfully treated with modafinil that improves significant sleepiness and is well-tolerated, without adverse effects. ${ }^{46} \mathrm{An}$ improvement of EDS has been reported in a case following treatment with tryptophan, possibly by consolidation of fragmented sleep. ${ }^{47}$

\section{Fragile $X$ Syndrome}

Fragile X syndrome (FXS) is one of the most common causes of MR; it is characterized by mild-to-severe intellectual disability, epilepsy, abnormalities in language and communication, unusual responses to sensory stimuli, stereotypic behaviors, hyperactivity, and deficits in visual-spatial skills, attention, and executive functioning. Distinctive physical features include an elongated face, large ears, and protruding jaw. ${ }^{48}$

Very few studies examined sleep disorders in patients with FXS: in two different studies Kronk et al reported a prevalence of 32 to $50 \%$ of significant sleep problems with the more frequent complaints represented by sleep onset difficulties and frequent awakenings during the night. ${ }^{49,50}$

Data obtained from the Fragile X Clinical and Research Consortium Database showed that $27 \%$ of parents report sleep problems. ${ }^{48}$ Also, OSA has been reported to be a frequent complain (from $7 \%$ to more than $30 \%$ of patients). ${ }^{51}$

A neurophysiologic study of nine patients with FXS showed reduced total sleep time, decreased REM sleep percentage, and an increase in the first REM latency and in stage 3 to 4 NREM percentage. ${ }^{52}$ Moreover, in the same study, an increase in twitch movements was observed during both stage 2 NREM and REM sleep. This may indicate a dysregulation of the cholinergic monoaminergic system during sleep, leading to an imbalance between the two neurochemically different mechanisms, which are known to be involved in other clinical manifestations of this genetic syndrome. ${ }^{53}$

Behavioral intervention for sleep problems (mainly extinction) may be effective in subjects with FXS reducing settling problems, night awakenings, and co-sleeping while early morning waking and night rocking do not improve. ${ }^{54}$

Recently, research has been focused on medical problems in FMR1 premutation carriers-FMR1 premutation occurs in 1:200 women and 1:400 men. ${ }^{55}$ An association between this condition and fragile $\mathrm{X}$ tremor and ataxia syndrome (FXTAS) has been reported, but sleep problems in this population are overlooked. Hamlin et al showed increased prevalence of OSA in premutation carriers with FXTAS and a significant odds 
ratio of sleep apnea for permutation carriers with FXTAS of approximately 3.4 times emerged. ${ }^{56}$

A recent study examined the relationship between fragile $\mathrm{X}$ premutation and restless legs syndrome (RLS)-individuals with premutation resulted 1.9 times as likely to meet criteria for RLS versus controls and premutation carriers with RLS experienced also significantly worse symptoms and higher scores at the International restless legs scale, insomnia severity scale, and Pittsburgh Sleep Quality Index. ${ }^{57}$

\section{Smith-Magenis Syndrome}

Smith-Magenis syndrome (SMS) is a rare, complex multisystemic disorder caused by a mutation or small interstitial deletion in a crucial transcriptional regulator gene of the mammalian circadian clock, RAI1 (retinoic acid induced) on chromosome $17 \mathrm{p} 11.2^{58}$; it occurs in $1: 25,000$ births, and is characterized by developmental delay with intellectual disability of variable degree, short stature, hoarse deep voice, obesity, scoliosis, distinctive facies (deep, close-set eyes, midfacial hypoplasia, cupid bow-shaped mouth, and broad, square-shaped face), and peripheral neuropathy. ${ }^{59}$

Maladaptive behaviors are typical of SMS-self-injury with low sensitivity to pain (hitting, biting, skin picking, inserting foreign objects into body orifices, and yanking nails); peculiar motor stereotypes, including upper body self-hugging, compulsive finger licking, and book or magazine page flipping; temper tantrums, oppositional defiant behaviors, and attention deficit/hyperactivity disorder (ADHD) and a 24-hour sleep disturbance.

The diagnosis of SMS is based on clinical recognition of this constellation of physical, developmental, and behavioral features in combination with a sleep disorder characterized by inverted circadian rhythm of melatonin secretion with peaks during the day. ${ }^{60}$

Sleep disorders characterize all ages of patients with SMSin infants' sleep disturbance is manifest by EDS or lethargy, as well as by decreased sleep duration. Older toddlers and children show fragmented and shortened sleep cycles, frequent and prolonged nocturnal awakenings, early morning awakening, EDS, daytime napping, snoring, and enuresis. Decreased nocturnal sleep, early awakenings, and daytime naps extend into adolescence. ${ }^{61}$

Data derived from actigraphy indicate a sleep disturbance that begins as early as 6 months of age during infancy, with fragmented sleep and reduced 24-hour sleep compared with healthy control subjects. This pattern persists in preschool (3 years), early school (5 years), and later school children (6-8 years) that sleep 1 to 2 hours less per 24 hours than healthy age-matched control children. Reduced 24hour sleep stems largely from the reduction of night sleep in all age groups. The sleep debt is compensated for by daytime napping. ${ }^{60}$

PSG studies revealed reduced sleep time in virtually all SMS patients; they showed an abnormally reduced latency for falling asleep during the daytime, a finding that is consistent with increased daytime napping; in addition, abnormalities of REM sleep have also been reported in 43 to $50 \%$ of patients with SMS. ${ }^{62}$
As somatic features like such as hypoplasia and obesity predispose patients with SMS to OSAS, also this disorder should be screened.

CRSD in SMS are thought to be related to the disturbed regulation of downstream circadian clock genes. ${ }^{58,63}$

A series of studies have found that $96 \%$ of SMS children had inverted endogenous melatonin secretion, peaking in the day rather than at night. ${ }^{64}$

In a small group of patients with SMS, oral acebutolol was given in the early morning to suppress the daytime melatonin secretion and was coupled with an evening dose of melatonin to improve sleep/wake complaints. ${ }^{65,66}$

\section{Rett Syndrome}

RS is a rare neurodevelopmental disorder associated, in 99\% of cases, with a de novo mutation of the MECP2 (methyl-CpG binding protein 2 ) gene on the $\mathrm{X}$ chromosome (Xq28) producing the MECP2 protein. $^{67}$ It affects mainly females with a prevalence of $1: 10,000$ to $20,000{ }^{68}$ It is the second most common cause of genetic intellectual disability in females (after DS) and is characterized by severe MR, autistic features, muscle hypotonia, deceleration of head growth, ventilatory abnormalities during wakefulness (alternating apnea and hyperventilation), and motor apraxia of unknown etiology. ${ }^{69}$

Epilepsy occurs in 50 to $90 \%$ of patients with RS, with drugresistant nocturnal seizures. ${ }^{70}$

Early studies on RS showed characteristic sleep dysfunctions, that are included as part of the diagnostic criteria for RS, represented by irregular sleep/wake patterns, excessive daytime naps, and problematic nighttime behaviors, such as, nocturnal laughter, bruxism, long spells of screaming and/or inconsolable crying, nocturnal seizures, sleep terrors, and sleep talking. ${ }^{71}$

Studies using polysomnography revealed alterations in sleep architecture such as lower sleep efficiency, long sleep-onset latency, and short total sleep time, increased wake after sleep onset (WASO), decreased REM sleep, fewer spindles, and K complexes similar to other forms of MR. ${ }^{72-74}$ Patients with RS commonly show irregular breathing during wakefulness consisting of episodes of hyperventilation interspersed by breath-holding spells, sometimes associated with severe oxygen desaturation. On the contrary, usually, the respiratory pattern during sleep is normal, although some central or obstructive events and irregular breathing in later stages of the disorder were also reported. ${ }^{73}$

A recent study showed, in a sample of more than 300 cases followed over 12 years, that the prevalence of any sleep disturbance was very high (more than $80 \%$ ) and decreased with age (less common in individuals older than 18 years) ${ }^{75}$ Night laughing represented the most frequent problem occurring in 60to $88 \%$ of younger girls followed by night screaming (less than 50\%). The authors also demonstrated that, after adjusting for age, a higher prevalence of night laughing was associated with a larger gene deletion in patients with RS.

Treatment strategies for respiratory disturbances included 1 to 2 hours per day of continuous positive airway pressure (CPAP) while awake. ${ }^{76}$ 
Behavioral insomnia and problematic nighttime behaviors in RS are usually treated with a combination of behavioral treatments (graduated extinction) and oral melatonin (range, $2.5-7.5 \mathrm{mg}$ ) that reduced mean sleep latency. ${ }^{77}$

\section{Sleep Abnormalities in Children with Autism Spectrum Disorders}

Sleep disorders and sleep alterations are common in children with ASDs, including Asperger syndrome. ${ }^{78}$ Studies based on parental surveys reported a 50 to $80 \%$ prevalence of sleep problems in children with ASD compared with a 9 to $50 \%$ prevalence rate in typically developing children. ${ }^{78-80}$ The degree of cognitive impairment or the ASD subtype do not seem to be correlated with the prevalence of sleep problems but, on the contrary, sleep disturbance in these children seems to be correlated with aggressive behavior, developmental regression, and internalizing problems. ${ }^{81}$ Sleep disorders were reported either in ASD children with severe MR or in high-functioning subjects, with intelligence quotients greater than $70 .{ }^{78}$

In a questionnaire-based study on 167 children with ASD, Liu et al revealed that approximately $86 \%$ of subjects had at least one sleep problem almost every day, including bedtime resistance (54\%), insomnia (56\%), parasomnias (53\%), sleepdisordered breathing (25\%), morning rise problems (45\%), and daytime sleepiness (31\%). ${ }^{82}$ Recently, it has been reported that more than $50 \%$ of 2 - to 5 -year-old children with ASD have at least one sleep problem, take more than 1 hour to fall asleep, and have long-lasting nocturnal awakenings. ${ }^{83}$ Furthermore, insomnia is 10 times more frequent in children with ASD and this symptom tends to persist until adolescence. ${ }^{84}$

Anxiety, autism symptom severity, and gastrointestinal problems were identified as the main risk factors for sleep disturbance in children with ASD. ${ }^{85}$

It is known that in ASD, there is an overall diminished pattern of nocturnal melatonin secretion or a delay in its secretion at night. Low levels of melatonin and/or its urinary metabolic derivatives correlate with sleep problems and autistic behaviors. ${ }^{86}$

Therefore, patients with ASD may be unable to fall asleep until late at night or may exhibit irregular sleep-wake rhythms with multiple naps distributed through day and night. $^{87}$

Emotional difficulties (e.g., separation anxiety) or communication difficulties that may preclude them from comprehending parental instructions about falling asleep may be the cause of insomnia. ${ }^{88}$ Also, daytime sleepiness was frequently observed in children with ASD-Bruni et al using the Pediatric Daytime Sleepiness Scale reported that $62 \%$ of children with Asperger syndrome exhibited scores $>16$, denoting excessive sleepiness. ${ }^{89}$

PSG studies in children with ASD have focused on abnormalities related to REM sleep (decreased quantity and reduced eye movement density during REM), decreased sleep spindles in Stage 2, increased stage 1, and decreased SWS. ${ }^{78,87}$

Few studies have tried to analyze the sleep microstructure to characterize better sleep of autistic children. Miano et al revealed that children with ASD, compared with typical developing children, showed a reduced total sleep, shorter REM latency, and lower CAP rate, mainly caused by a reduction of A1 CAP subtypes. ${ }^{87}$ Bruni et al reported that in children with Asperger syndrome sleep architecture does not differ from that of children with autism, but, on the contrary, sleep microstructure presents several differences-increased CAP rate in SWS and a decrease in sleep stage 2 , higher percentage of A1 subtypes, and lower $A 2 \% .{ }^{89}$

A PSG study of two consecutive nights on ASD children showed that the ASD poor sleepers differed significantly from the ASD good sleepers and normal children, having lower sleep efficiency, and prolonged sleep latency. ${ }^{90}$

The management of sleep disturbance in ASD children depends on the type of sleep disorder, but, in general, behavioral therapy associated to melatonin supplementation is the most used.

Behavioral therapy: Children with ASD are very sensitive to noise, music, and daytime routine; thus, consistent sleeping environment and routine that do not vary from one night to another help the child relax down to sleep. Also, the avoidance of exciting or mentally stimulating activities in the hours before bedtime is important. ${ }^{81}$ Behavioral insomnia and sleep onset association disorder require parents to practice techniques of behavioral extinction and/or decreasing the duration of the afternoon nap. ${ }^{91}$

Melatonin: Melatonin given its endogenous deficiency in a significant percentage of children with ASD, supplementation with melatonin is suggested for difficulties initiating and maintaining sleep. The time of management is generally 5 to 6 hours before the desired bedtime, on an empty stomach in doses of 1 to $6 \mathrm{mg} .^{88,92,93}$ In a study on 51 patients (aged 218 years) with NDDs, melatonin was provided 20 to 30 minutes before the desired bedtime and improved sleep in 47 of 51 subjects-both sleep latency and total sleep time improved by approximately 30 minutes and no significant treatment-related side effects were reported. ${ }^{94}$

\section{Sleep Abnormalities in Children with Cerebral Palsy}

The appropriate diagnosis and treatment of sleep problems in children with cerebral palsy (CP) is extremely important, because of their consequences on both child and family. ${ }^{2}$

$\mathrm{CP}$ is defined as a group of disorders of movement and posture causing activity limitations that can be attributed to nonprogressive disturbances that occur in the developing fetal or infant brain. The motor disorders of $\mathrm{CP}$ are often accompanied by disturbances of sensation, cognition, communication, perception, behavior, and/or concurrent epilepsy.

$\mathrm{CP}$ is estimated to occur in 1.5 to 2.5 children per 1,000 live births in developed countries and is the most common cause of childhood physical impairment. ${ }^{95}$

In children with $\mathrm{CP}$, sleep may be affected by the following several factors: muscle spasms, muscle-skeletal pain, and decreased ability to change body position during the night may all contribute to sleep difficulties. Epilepsy is likely to 
predispose to sleep disorders, and the antiepileptic drugs can cause daytime sleepiness and deeply modify sleep architecture. Approximately 20 to $50 \%$ of children with CP have a cortical visual impairment, which can affect the timing and maintenance of sleep through an effect on melatonin secretion and the lack of light perception, resulting in a free running circadian rhythm. ${ }^{96}$

The most frequent subtypes of sleep disorders in this population are disorders of initiation and maintenance of sleep, sleep-wake transition disorders, parasomnias, and OSA. ${ }^{97}$

The incidence and severity of motor impairment and comorbidities, including sleep disorders, is partially related to severity of brain damage and subtype of $\mathrm{CP}$, where bilateral spastic $\mathrm{CP}$ or quadriplegia are the most impairing for the quality of life and of sleep. A recent questionnaire study showed that sleep disturbances were significantly associated with the presence of quadriplegia, of epilepsy, of internalizing and total CBCL score, and of level V on Gross Motor Function Classification System (GMFCS). ${ }^{98}$

Anatomical factors such as pharyngeal hypotonia, adenotonsillar hypertrophy, glossoptosis, and disproportionate midface anatomy or mandibular alterations, together with abnormal central control of respiration can contribute to the obstructive disorder, causing sleep fragmentation and intermittent hypoxemia. Moreover, patients with CP suffer from gastroesophageal reflux, which may determine sleep fragmentation. Scoliosis and restricted lung volumes may aggravate the risk of sleep-related hypoventilation.

A questionnaire-based survey of $233 \mathrm{CP}$ children investigated the prevalence of respiratory disturbances during sleep; habitual snoring was reported in $63 \%$ and sleep apnea in $19.7 \%$. In 48 of these children whose questionnaires revealed habitual snoring and sleep apnea, a screening sleep study using pulse oximetry showed that $27 \%$ of the children had an apnea-hypopnea index (AHI) > 5 and 58\% had a level of oxygen saturation lower than $85 \% .{ }^{99}$

Wiggs and Stores found a significant association between the diagnosis of $\mathrm{CP}$ and chronic sleep problems in a population of motor-disabled children ${ }^{100}$; in a questionnaire-based survey, $23 \%$ of the children with $\mathrm{CP}$ had an abnormal total sleep score and $44 \%$ had at least one clinically significant sleep disorder. Furthermore, $14.5 \%$ of the 173 children with $\mathrm{CP}$ had a pathologic score for sleep-related breathing disorders at the Sleep Disturbance Scale for Children, but the authors did not consider the presence of MR and behavioral problems, which are known to have a strong influence on sleep. ${ }^{97}$

One of the first reports exploring sleep organization in children with CP showed that 12 of 23 patients exhibited some abnormal sleep EEG pattern, such as absence of EEG characteristics indicative of wakefulness, NREM sleep and REM sleep, absence of REM sleep, extremely low incidence of sleep spindles or the presence of "extreme spindles" or abnormally high percentage of wake after sleep onset. ${ }^{101}$

An old study on 56 children with persistent snoring and OSA, 16 of whom were neurologically abnormal (two of them with $\mathrm{CP}$ ), showed that neurologically abnormal children had significantly increased obstructive apnea indices, increased desaturation events, and lower mean arousal indices compared with their neurologically normal OSA peers. ${ }^{102}$

A more recent questionnaire-based study of children with $\mathrm{CP}$ seems to indicate that this population is indeed at higher risk for different sleep disorders: more than $40 \%$ of children with $\mathrm{CP}$ presented with at least one sleep disorder, versus $5 \%$ in the normal population, with a strong association between the SDSC total scores, epilepsy, MR and level 5 on the GMFCS. $^{103}$

No sleep interventions specifically designed to improve sleep of children with $\mathrm{CP}$ are reported in the literature, and only melatonin remains a commonly prescribed drug for disturbed sleep in children with neurologic dysfunction.

Treatment of OSA with adenotonsillectomy or CPAP may improve sleep and quality of life in children with $\mathrm{CP}^{104}$; surgical techniques for the treatment of sleep respiratory disturbances have been used in several patients with $\mathrm{CP}$ or anoxic brain injury with documented obstructive sleep apnea; improvement of respiratory symptoms was achieved in most of patients treated with a significant reduction of AHI. ${ }^{105,106}$

Comorbid epilepsy in these children might also affect sleep through a defect in sleep regulation, presence of nocturnal seizures, and side effects of the antiepileptic medication. ${ }^{107}$ Furthermore, spasticity, contractures, and movement limitations, with associated pain may adversely affect sleep. The reduction of spasticity in CP patients can lead to an improvement of sleep maintenance trough reduction of pain, and reduction of respiratory muscle spasticity, ameliorating the respiratory function during sleep. Intrathecal baclofen therapy in a patient with mixed spastic athetoid quadriparetic $\mathrm{CP}$ and sleep apnea, requiring nightly continuous positive airway pressure, determined a reduction of spasticity, as well as an improvement of sleep apnea. ${ }^{108}$

Published data about sleep disorders in CP were mainly obtained by using screening questionnaires; for this reason, additional studies with structured interview or objective assessment may provide adequate information on sleep disorders and allow us to identify effective treatments.

\section{Therapeutic Approach to Sleep Disturbances in Children with Neurodevelopmental Disabilities}

Most reviews of the pharmacological treatment of sleep disorders in children with NDDs focused mainly on insomnia and not on the other frequent comorbid disorders, such as respiratory sleep disturbances, rhythmic movement disorders, RLS, or periodic limb movements during sleep.

It should be emphasized that each developmental disorder could be characterized by a specific type of sleep disorder that should be accurately diagnosed to carry out the correct treatment. Therefore, after an accurate history and physical examination, a PSG study is mandatory if there is a reasonable suspect of the presence of one of the sleep disorders aforementioned.

Besides the treatment of the specific sleep disorders, an overview of the cognitive behavioral treatment and of the 
most commonly used drugs for sleep disturbances is reported in the following paragraphs.

\section{Behavioral Treatment}

After excluding medical contributors and other primary sleep disorders, that is, epilepsy, gastroesophageal reflux, iron deficiency, OSA, periodic limb movements or CRSD, parentbased education, and behavioral interventions are the firstline treatment of insomnia in NDDs.

Although genetic and/or epigenetic abnormalities in sleep/ wake regulation predispose patients with NDDs to insomnia, poor sleep hygiene, negative associations and lack of limit setting, contribute to maintaining sleep disruption. Therefore, all behavioral techniques aim to promote self-soothing skills that allow the child to fall and return to sleep independently.

Parents are fundamental active figures to implement behavioral treatment strategies, such as, promoting a dark, quiet, relatively cool, nonstimulating environment, and poor of visual and auditory stimuli (such as electronic devices); parents should establish a successful and consistently followed bedtime routine and maintain a consistent sleep/wake schedule, avoiding the child to go to bed earlier or sleeping later due to lost sleep. ${ }^{4}$

In the case of disruptive behaviors, parents must avoid responding because this would give a negative reward to such behaviors. In the case of difficulty to ignore the child, parents may establish a minimal checking protocol, periodically observing and reassuring the child if he/she accepts to stay quiet in bed. This technique should be reapplied for nighttime awakenings.

\section{Drugs Commonly Used for Sleep Disturbances in Children with Neurodevelopmental Disabilities}

If insomnia in a child with NDDs remains problematic after cognitive behavioral therapy, initiating sleep-promoting agents while continuing behavioral intervention is recommended.
In a recent survey study by Owens et al, more than onethird of 1,273 child psychiatrist treating insomnia in children with psychiatric disorders and NDDs reported that they treated insomnia with medication at least half of the time in patients with ADHD, ASD, and with mental retardation/ developmental disabilities (MR/DD). Moreover, they reported to treat $17 \%$ of preschoolers and at least one-quarter of school-aged and adolescent patients. Overall, 96\% of respondents recommended at least one medication in a typical month, and $88 \%$ recommended an over-the-counter medication. 109

In general, respondents were more likely to use herbal preparations in children with anxiety or mood disorders than in children with NDDs or ADHD; melatonin was recommended by more than one-third of respondents (39\%) although it is unclear whether it was being used primarily at bedtime for its mild hypnotic effects or as a chronobiotic. The physicians recommended for sleep disorders (mainly insomnia) nonprescription antihistamines in $69 \%$ and $\alpha$ agonists in $67 \%$ of children with MR/DD. Trazodone was the second most frequently prescribed medication for children with MR/DD (66\%) while sedating antidepressants were used in $75.5 \%$ of MR cases. Atypical antipsychotics were used by more than half of the respondents in children with MR/DD (52\%) and benzodiazepines were used in $21.6 \%$ of cases of MR/DD. Tryciclic antidepressants as a class were also used for children in these diagnostic groups (25.5\%).

Despite the widespread use of pharmacological treatment, the lack of well designed, controlled studies concerning the efficacy, tolerability, dosing, and safety profile of hypnotic medications in children are still needed. - Table 1 summarizes the most common drugs used for insomnia in children with NDDs.

\section{Melatonin}

Melatonin ( $\mathrm{N}$-acetyl-5-methoxytryptamine) is a chronobiotic drug crucial for the regulation of the sleep-wake cycle. In

Table 1 Selected medications for the treatment of insomnia in subjects with NDDs

\begin{tabular}{|l|l|l|}
\hline Medication & Dosage & Indications \\
\hline Dyphenidramine & $0.5 \mathrm{mg} / \mathrm{kg}$ & $\begin{array}{l}\text { Primary insomnia with delayed sleep onset and/or frequent nocturnal } \\
\text { awakenings }\end{array}$ \\
\hline Hydroxizine & $1 \mathrm{mg} / \mathrm{kg}$ & $\begin{array}{l}\text { Primary insomnia with delayed sleep onset and/or frequent nocturnal } \\
\text { awakenings }\end{array}$ \\
\hline Niaprazine & $1 \mathrm{mg} / \mathrm{kg}$ & $\begin{array}{l}\text { Primary insomnia with delayed sleep onset and/or frequent nocturnal } \\
\text { awakenings }\end{array}$ \\
\hline Melatonin & $0.5-6 \mathrm{mg}$ & Circadian rhythm disorders \\
\hline Zolpidem & $5-10 \mathrm{mg}$ & Primary insomnia with delayed sleep onset \\
\hline Trazodone & $50-150 \mathrm{mg}$ & Primary insomnia; frequent nocturnal awakenings \\
\hline Clonidine & $0.05-0.1 \mathrm{mg}$ & ADHD, disruptive behavior disorders \\
\hline Gabapentin & $300-900 \mathrm{mg}$ & Restless legs syndrome, epilepsy, resistant sleep onset insomnia \\
\hline Clonazepam & $0.25-0.5 \mathrm{mg}$ & $\begin{array}{l}\text { Epilepsy, restless legs syndrome, resistant sleep onset insomnia, bruxism, } \\
\text { rhythmic movement disorder }\end{array}$ \\
\hline
\end{tabular}

Abbreviations: ADHD, attention deficit/hyperactivity disorder; NDDs, neurodevelopmental disabilities. 
older children and adults, its production and secretion begin in the evening and peak during the night between 2:00 and 4:00 Am; its production and release are inhibited by light. There is now a greater understanding that low doses $(0.5 \mathrm{mg})$ can be effective for some children, with diminishing benefit with doses exceeding $6 \mathrm{mg}$ and, unlike traditional hypnotics such as chloral hydrate and the benzodiazepines, melatonin does not affect sleep architecture. ${ }^{110}$

Melatonin is increasingly prescribed to many children, both with and without NDDs and using a wide range of doses, demonstrating efficacy in improving sleep quality, by reducing sleep-onset latency or slightly increasing total sleep time. These effects have been observed in typically developing children with delayed sleep-phase syndrome, but they appear to be stronger in children with visual impairment, NDDs, attention deficit, and ASD. ${ }^{111-113}$

A recent large clinical trial confirmed the efficacy of melatonin in the treatment of sleep impairment in children with NDDs, using different doses, ranging from 0.5 to $12 \mathrm{mg}$; the main effects of melatonin were reduced sleep latency (from 102 to 55 minutes in 12 weeks) and increased total sleep time (40 minutes). ${ }^{114}$

Mild side effects such as headache, confusion, dizziness, cough, and rashes have been reported with the use of melatonin, but systematic reviews and meta-analyses suggest that there are no significant adverse side effects. ${ }^{112,113}$

\section{Gabapentin}

The Food and Drug Administration (FDA) approved gabapentin for treatment of partial seizures in 1993. It was originally designed as a precursor of $\gamma$-amino butyric acid (GABA) that easily enters the blood-brain barrier, and increases brain synaptic GABA. It has been approved for the treatment of neuropathic pain and RLS in addition to its original purpose as an anticonvulsant medication. However, its precise pharmacological mechanism in humans remains unknown.

In an open-label study in adults, gabapentin was found to improve sleep quality in patients with primary insomnia, as it increases slow-wave sleep and sleep efficiency; it also decreases WASO and spontaneous arousal. ${ }^{115}$

In a recent case series, gabapentin was found to be a safe and a well-tolerated treatment for sleep-onset and sleep maintenance insomnia in the majority of treated children, including those with neurodevelopmental or neuropsychiatric disorders. Overall, 78\% of children showed improvement in sleep (as reported by parents). Furthermore, this beneficial response was noted at doses of 5 to $15 \mathrm{mg} / \mathrm{kg}$ orally at bedtime, much less than the recommended dose to treat epileptic seizures ( $40 \mathrm{mg} / \mathrm{kg}$ divided three times daily). ${ }^{116}$

Gabapentin may have the added benefit of treating also sleep maintenance insomnia in children with NDDs, although larger controlled trials will be needed to establish its efficacy.

\section{Clonidine}

Clonidine is a central and peripheral $\alpha$-adrenergic agonist that acts on presynaptic neurons and inhibits noradrenergic release and transmission, approved by the FDA for the treatment of hypertension. Clonidine received notoriety for being prescribed as a sleep aid in children, but currently, there are no well-controlled studies that address the effects of clonidine in children with sleep problems. ${ }^{117}$

It is hypothesized that clonidine produces sedation via decrease in norepinephrine via negative feedback by agonism of the $\alpha_{2}$-adrenergic receptors at the level of the locus coeruleus, which would increase REM sleep; there appears to be variable effects on sleep, which are dose dependent. Administration of low doses of clonidine (range, 0.025-0.05 $\mathrm{mg}$ ) has little effect on sleep and can either increase or decrease the duration of REM sleep. At medium-to-high doses (range, $0.1-0.3 \mathrm{mg}$ ), clonidine appears to have postsynaptic activity on the $\alpha_{2}$-adrenergic receptors, which results in decrease of acetylcholine, which increases REM latency, stage 2 sleep, and slow-wave sleep. ${ }^{118}$

Clonidine presents the advantage of transdermal administration and the central nervous system side effects are typically less evident with the transdermal patch. The most commonly reported side effects of clonidine include drowsiness, transient sedation, headache, dizziness, fatigue, somnolence, insomnia, hypotension, and bradycardia. These side effects commonly subside or decrease variably over time and are thought to be dose dependent.

Ingrassia Turk in a retrospective chart review found clonidine to be an effective therapeutic intervention for alleviating sleep disturbances in six children, whose ages ranged from 6 to 14 years. Dose titration began at $0.05 \mathrm{mg}$ and was gradually titrated up to $0.1 \mathrm{mg}$ at bedtime. No severe side effects were reported. ${ }^{119}$

In a recent open label retrospective review, 19 children with ASD were treated with oral clonidine (range, $0.1-0.2 \mathrm{mg}$ ) 30 minutes before bed-reduced sleep latency and lessened nocturnal awakenings; this is especially important in children with ASD who are overly aroused or mildly anxious at bedtime. $^{120}$

Moreover, Hollway et al performed a vast literature search, and clonidine was reported to be effective in children who experienced sleep disturbances with comorbid ASD and other neurodevelopmental disorders with behavioral problems at doses ranging from 0.05 to $0.225 \mathrm{mg} / \mathrm{d}$. ${ }^{121}$

A small number of clonidine overdoses have been reported with resulting fatality and there is some evidence that the clonidine overdose may be becoming more frequent ${ }^{122}$; however, generally, clonidine is well tolerated in children and has been efficacious in treating pediatric insomnia.

Future trials with clonidine in children should be fully controlled with objective measures, including PSG recordings, to determine its effectiveness and safety.

\section{Clonazepam}

Benzodiazepines bind to the benzodiazepine subunit of the GABA chloride receptor complex, facilitating the action of the inhibitory neurotransmitter GABA. These hypnotics have long been the first choice treatment for insomnia in adults, but they raise concerns about cognitive impairment, rebound insomnia, and the potential risk for dependence. These concerns and little evidence-based data availability in the pediatric population, contribute to limit their use in children. 
Clonazepam is a benzodiazepine with strong anticonvulsant, muscle relaxant, and sedative properties.

Three uncontrolled studies of children with parasomnias and secondary sleep disturbance showed that clonazepam improved (1) nocturnal tongue biting, (2) REM sleep behavior disorder, and/or (3) periodic limb movement disorder (PLMD), with mild and tolerable side effects. ${ }^{123-125}$

In a fully controlled study of adults without NDDs, the investigators found that clonazepam significantly improved PLMD and decreased the number of nighttime arousals. $^{126}$

From the available data, clonazepam may represent a treatment option in children with arousal disorders (parasomnias) or PLMD/RLS, but future trials focused on objective sleep measures and safety issues are needed.

\section{Conclusions}

Insomnia in children with NDDs is much more prevalent than in typically developing children, has multifactorial origin, and tends to be chronic. Chronic sleep deprivation compromises quality of life of both children and families and is associated with poorer developmental outcome, overweight, and behavioral disturbances.

In some NDDs, problematic sleep is a phenotypic characteristic of a particular disorder and can be a clinical clue to the diagnosis.

The medical approach should follow the pathway of sleep medicine, examining medical and psychiatric contributing factors, primary sleep disorders and maladaptive behaviors related to sleep.

Some of the following treatment options are available: behavioral treatment strategies trough the parents, circadian rhythm regulation trough melatonin, and pharmacological treatment or specific treatment in the case of obstructive sleep apnea.

Awareness of features of sleep disorders in patients with NDDs is fundamental for the appropriate recognition and effective treatment of these often overlooked aspect.

Despite the widespread use of pharmacological treatment, the lack of well designed, controlled studies concerning the efficacy, tolerability, dosage, and safety profile of hypnotic medications in children raise the need of further research in this field of sleep medicine.

\section{References}

1 Zucconi M, Bruni O. Sleep disorders in children with neurologic diseases. Semin Pediatr Neurol 2001;8(4):258-275

2 Zucconi M, Bruni O. Sleep in children with neurologic disease. In: Marcus C LG, Caroll J, Donnelly D, eds. Sleep in Children. Abingdon, Oxon: Taylor \& Francis Group; 2008:261-296

3 Stores G. Sleep studies in children with a mental handicap. J Child Psychol Psychiatry 1992;33(8):1303-1317

4 Grigg-Damberger M, Ralls F. Treatment strategies for complex behavioral insomnia in children with neurodevelopmental disorders. Curr Opin Pulm Med 2013;19(6):616-625

5 Quine L. Sleep problems in children with mental handicap.J Ment Defic Res 1991;35(Pt 4):269-290
6 Okawa MSH. Sleep disorders in mentally retarded and brainimpaired children. In: CG, ed. Sleep and its Disorders in Children. New York, NY: Raven Press; 1987:269-290

7 Dyken ME, Lin-Dyken DC, Poulton S, Zimmerman MB, Sedars E. Prospective polysomnographic analysis of obstructive sleep apnea in down syndrome. Arch Pediatr Adolesc Med 2003;157(7): 655-660

$8 \mathrm{Ng} \mathrm{DK}$, Hui HN, Chan $\mathrm{CH}$, et al. Obstructive sleep apnoea in children with Down syndrome. Singapore Med J 2006;47(9): 774-779

9 Hoffmire CA, Magyar CI, Connolly HV, Fernandez ID, van Wijngaarden E. High prevalence of sleep disorders and associated comorbidities in a community sample of children with Down syndrome. J Clin Sleep Med 2014;10(4):411-419

10 Allanson JE, O'Hara P, Farkas LG, Nair RC. Anthropometric craniofacial pattern profiles in Down syndrome. Am J Med Genet 1993; 47(5):748-752

11 Aboussouan LS, O'Donovan PB, Moodie DS, Gragg LA, Stoller JK. Hypoplastic trachea in Down's syndrome. Am Rev Respir Dis 1993;147(1):72-75

12 Ferri R, Curzi-Dascalova L, Del Gracco S, Elia M, Musumeci SA, Pettinato S. Heart rate variability and apnea during sleep in Down's syndrome. J Sleep Res 1998;7(4):282-287

13 McKay SM, Angulo-Barroso RM. Longitudinal assessment of leg motor activity and sleep patterns in infants with and without Down syndrome. Infant Behav Dev 2006;29(2):153-168

14 Ashworth A, Hill CM, Karmiloff-Smith A, Dimitriou D. Cross syndrome comparison of sleep problems in children with Down syndrome and Williams syndrome. Res Dev Disabil 2013;34(5):1572-1580

15 Breslin JH, Edgin JO, Bootzin RR, Goodwin JL, Nadel L. Parental report of sleep problems in Down syndrome. J Intellect Disabil Res 2011;55(11):1086-1091

16 Carter M, McCaughey E, Annaz D, Hill CM. Sleep problems in a Down syndrome population. Arch Dis Child 2009;94(4):308-310

17 Diomedi M, Curatolo P, Scalise A, Placidi F, Caretto F, Gigli GL. Sleep abnormalities in mentally retarded autistic subjects: Down's syndrome with mental retardation and normal subjects. Brain Dev 1999;21(8):548-553

18 Clausen J, Sersen EA, Lidsky A. Sleep patterns in mental retardation: Down's syndrome. Electroencephalogr Clin Neurophysiol 1977;43(2):183-191

19 Bull MJ. Committee on Genetics. Health supervision for children with Down syndrome. Pediatrics 2011;128(2):393-406

20 Bird LM. Angelman syndrome: review of clinical and molecular aspects. Appl Clin Genet 2014;7:93-104

21 Kyllerman M. Angelman syndrome. Handb Clin Neurol 2013; 111:287-290

22 Clayton-Smith J, Laan L. Angelman syndrome: a review of the clinical and genetic aspects. J Med Genet 2003;40(2):87-95

23 Williams CA, Beaudet AL, Clayton-Smith J, et al. Angelman syndrome 2005: updated consensus for diagnostic criteria. Am J Med Genet A 2006;140(5):413-418

24 Pelc K, Cheron G, Boyd SG, Dan B. Are there distinctive sleep problems in Angelman syndrome? Sleep Med 2008;9(4): 434-441

25 Miano S, Bruni O, Leuzzi V, Elia M, Verrillo E, Ferri R. Sleep polygraphy in Angelman syndrome. Clin Neurophysiol 2004; 115(4):938-945

26 Bruni O, Ferri R, D’Agostino G, Miano S, Roccella M, Elia M. Sleep disturbances in Angelman syndrome: a questionnaire study. Brain Dev 2004;26(4):233-240

27 Didden R, Korzilius H, Smits MG, Curfs LM. Sleep problems in individuals with Angelman syndrome. Am J Ment Retard 2004; 109(4):275-284

28 Laan LA, den Boer AT, Hennekam RC, Renier WO, Brouwer OF. Angelman syndrome in adulthood. Am J Med Genet 1996;66(3): 356-360 
29 Conant KD, Thibert RL, Thiele EA. Epilepsy and the sleep-wake patterns found in Angelman syndrome. Epilepsia 2009;50(11): 2497-2500

30 Walz NC, Beebe D, Byars K. Sleep in individuals with Angelman syndrome: parent perceptions of patterns and problems. Am J Ment Retard 2005;110(4):243-252

31 Takaesu Y, Komada Y, Inoue Y. Melatonin profile and its relation to circadian rhythm sleep disorders in Angelman syndrome patients. Sleep Med 2012;13(9):1164-1170

32 Miano S, Bruni O, Elia M, Musumeci SA, Verrillo E, Ferri R. Sleep breathing and periodic leg movement pattern in Angelman Syndrome: a polysomnographic study. Clin Neurophysiol 2005; 116(11):2685-2692

33 Summers JA, Lynch PS, Harris JC, Burke JC, Allison DB, Sandler L. A combined behavioral/pharmacological treatment of sleep-wake schedule disorder in Angelman syndrome. J Dev Behav Pediatr 1992;13(4):284-287

34 Allen KD, Kuhn BR, DeHaai KA, Wallace DP. Evaluation of a behavioral treatment package to reduce sleep problems in children with Angelman Syndrome. Res Dev Disabil 2013;34(1): 676-686

35 Braam W, Didden R, Smits MG, Curfs LM. Melatonin for chronic insomnia in Angelman syndrome: a randomized placebo-controlled trial. J Child Neurol 2008;23(6):649-654

36 Donaldson MD, Chu CE, Cooke A, Wilson A, Greene SA, Stephenson JB. The Prader-Willi syndrome. Arch Dis Child 1994;70(1): 58-63

37 Kaplan J, Fredrickson PA, Richardson JW. Sleep and breathing in patients with the Prader-Willi syndrome. Mayo Clin Proc 1991; 66(11):1124-1126

38 State MW, Dykens EM. Genetics of childhood disorders: XV. Prader-Willi syndrome: genes, brain, and behavior. J Am Acad Child Adolesc Psychiatry 2000;39(6):797-800

39 Bruni O, Verrillo E, Novelli L, Ferri R. Prader-Willi syndrome: sorting out the relationships between obesity, hypersomnia, and sleep apnea. Curr Opin Pulm Med 2010;16(6):568-573

40 Verrillo E, Bruni O, Franco P, et al. Analysis of NREM sleep in children with Prader-Willi syndrome and the effect of growth hormone treatment. Sleep Med 2009;10(6):646-650

41 Nixon GM, Brouillette RT. Sleep and breathing in Prader-Willi syndrome. Pediatr Pulmonol 2002;34(3):209-217

42 Cohen M, Hamilton J, Narang I. Clinically important age-related differences in sleep related disordered breathing in infants and children with Prader-Willi Syndrome. PLoS ONE 2014;9(6): e101012

43 Sedky K, Bennett DS, Pumariega A. Prader Willi syndrome and obstructive sleep apnea: co-occurrence in the pediatric population. J Clin Sleep Med 2014;10(4):403-409

44 Nixon GM, Rodda CP, Davey MJ. Longitudinal association between growth hormone therapy and obstructive sleep apnea in a child with Prader-Willi syndrome. J Clin Endocrinol Metab 2011;96(1): 29-33

45 Berini J, Spica Russotto V, Castelnuovo P, et al; Genetic Obesity Study Group of the Italian Society of Pediatric Endocrinology and Diabetology (ISPED). Growth hormone therapy and respiratory disorders: long-term follow-up in PWS children. J Clin Endocrinol Metab 2013;98(9):E1516-E1523

46 De Cock VC, Diene G, Molinas C, et al. Efficacy of modafinil on excessive daytime sleepiness in Prader-Willi syndrome. Am J Med Genet A 2011;155A(7):1552-1557

47 Lee YJ, Marcu S, Berall G, Shapiro CM. Tryptophan for the treatment of excessive daytime sleepiness in Prader-Willi syndrome. Indian Pediatr 2011;48(4):319-321

48 Kidd SA, Lachiewicz A, Barbouth D, et al. Fragile X syndrome: a review of associated medical problems. Pediatrics 2014;134(5): 995-1005

49 Kronk R, Bishop EE, Raspa M, Bickel JO, Mandel DA, Bailey DB Jr. Prevalence, nature, and correlates of sleep problems among children with fragile $\mathrm{X}$ syndrome based on a large scale parent survey. Sleep 2010;33(5):679-687

50 Kronk R, Dahl R, Noll R. Caregiver reports of sleep problems on a convenience sample of children with fragile X syndrome. Am J Intellect Dev Disabil 2009;114(6):383-392

51 Tirosh E, Borochowitz Z. Sleep apnea in fragile X syndrome. Am J Med Genet 1992;43(1-2):124-127

52 Musumeci SA, Elia M, Ferri R, Scuderi C, Del Gracco S. Evoked spikes and giant somatosensory evoked potentials in a patient with fragile-X syndrome. Ital J Neurol Sci 1994;15(7): 365-368

53 Brown WT, Jenkins E, Neri G, et al. Conference report: Fourth International Workshop on the fragile $\mathrm{X}$ and $\mathrm{X}$-linked mental retardation. Am J Med Genet 1991;38(2-3):158-172

54 Weiskop S, Richdale A, Matthews J. Behavioural treatment to reduce sleep problems in children with autism or fragile $\mathrm{X}$ syndrome. Dev Med Child Neurol 2005;47(2):94-104

55 Tassone F, Iong KP, Tong TH, et al. FMR1 CGG allele size and prevalence ascertained through newborn screening in the United States. Genome Med 2012;4(12):100

56 Hamlin A, Liu Y, Nguyen DV, Tassone F, Zhang L, Hagerman RJ. Sleep apnea in fragile $X$ premutation carriers with and without FXTAS. Am J Med Genet B Neuropsychiatr Genet 2011;156B(8): 923-928

57 Summers SM, Cogswell J, Goodrich JE, et al. Prevalence of restless legs syndrome and sleep quality in carriers of the fragile $\mathrm{X}$ premutation. Clin Genet 2014;86(2):181-184

58 Williams SR, Zies D, Mullegama SV, Grotewiel MS, Elsea SH. SmithMagenis syndrome results in disruption of CLOCK gene transcription and reveals an integral role for RAI1 in the maintenance of circadian rhythmicity. Am J Hum Genet 2012;90(6):941-949

59 Smith ACM, Boyd KE, Elsea SH, et al. Smith-Magenis Syndrome. 2001 Oct 22 [Updated 2012 Jun 28]. In: Pagon RA, Adam MP, Ardinger $\mathrm{HH}$, et al., editors. GeneReviews ${ }^{\circledR}$ [Internet]. Seattle, WA: University of Washington; 1993-2015. Available at: http:// www.ncbi.nlm.nih.gov/books/NBK1310/

60 Gropman AL, Duncan WC, Smith AC. Neurologic and developmental features of the Smith-Magenis syndrome (del 17p11.2). Pediatr Neurol 2006;34(5):337-350

61 Smith AC, Dykens E, Greenberg F. Sleep disturbance in SmithMagenis syndrome (del 17 p11.2). Am J Med Genet 1998;81(2): 186-191

62 Potocki L, Glaze D, Tan DX, et al. Circadian rhythm abnormalities of melatonin in Smith-Magenis syndrome. J Med Genet 2000; 37(6):428-433

63 Nováková M, Nevsímalová S, Príhodová I, Sládek M, Sumová A. Alteration of the circadian clock in children with Smith-Magenis syndrome. J Clin Endocrinol Metab 2012;97(2):E312-E318

64 De Leersnyder H. Smith-Magenis syndrome. Handb Clin Neurol 2013;111:295-296

65 Carpizo R, Martínez A, Mediavilla D, González M, Abad A, Sánchez-Barceló EJ. Smith-Magenis syndrome: a case report of improved sleep after treatment with beta1-adrenergic antagonists and melatonin. J Pediatr 2006;149(3):409-411

66 De Leersnyder $\mathrm{H}$. Inverted rhythm of melatonin secretion in Smith-Magenis syndrome: from symptoms to treatment. Trends Endocrinol Metab 2006;17(7):291-298

67 Amir RE, Van den Veyver IB, Wan M, Tran CQ, Francke U, Zoghbi HY. Rett syndrome is caused by mutations in X-linked MECP2, encoding methyl-CpG-binding protein 2. Nat Genet 1999;23(2): 185-188

68 Perry A. Rett syndrome: a comprehensive review of the literature. Am J Ment Retard 1991;96(3):275-290

69 Hagberg B. Clinical manifestations and stages of Rett syndrome. Ment Retard Dev Disabil Res Rev 2002;8(2):61-65

70 Dolce A, Ben-Zeev B, Naidu S, Kossoff EH. Rett syndrome and epilepsy: an update for child neurologists. Pediatr Neurol 2013; 48(5):337-345 
71 Young D, Nagarajan L, de Klerk N, Jacoby P, Ellaway C, Leonard H. Sleep problems in Rett syndrome. Brain Dev 2007;29(10): 609-616

72 Glaze DG, Frost JD Jr, Zoghbi HY, Percy AK. Rett's syndrome: characterization of respiratory patterns and sleep. Ann Neurol 1987;21(4):377-382

73 Marcus CL, Carroll JL, McColley SA, et al. Polysomnographic characteristics of patients with Rett syndrome. J Pediatr 1994; 125(2):218-224

74 Nomura Y, Segawa M. Natural history of Rett syndrome. J Child Neurol 2005;20(9):764-768

75 Wong K, Leonard H, Jacoby P, Ellaway C, Downs J. The trajectories of sleep disturbances in Rett syndrome. J Sleep Res 2015;24(2): 223-233

76 Julu PO, Witt Engerström I, Hansen S, Apartopoulos F, Engerström B; ESSRA group. Treating hypoxia in a feeble breather with Rett syndrome. Brain Dev 2013;35(3):270-273

77 McArthur AJ, Budden SS. Sleep dysfunction in Rett syndrome: a trial of exogenous melatonin treatment. Dev Med Child Neurol 1998;40(3):186-192

78 Richdale AL, Schreck KA. Sleep problems in autism spectrum disorders: prevalence, nature, \& possible biopsychosocial aetiologies. Sleep Med Rev 2009;13(6):403-411

79 Allik H, Larsson JO, Smedje H. Insomnia in school-age children with Asperger syndrome or high-functioning autism. BMC Psychiatry 2006;6:18

80 Giannotti F, Cortesi F, Cerquiglini A, et al. An investigation of sleep characteristics, EEG abnormalities and epilepsy in developmentally regressed and non-regressed children with autism. J Autism Dev Disord 2008;38(10):1888-1897

81 Kotagal S, Broomall E. Sleep in children with autism spectrum disorder. Pediatr Neurol 2012;47(4):242-251

82 Liu X, Hubbard JA, Fabes RA, Adam JB. Sleep disturbances and correlates of children with autism spectrum disorders. Child Psychiatry Hum Dev 2006;37(2):179-191

83 Cortesi F, Giannotti F, Ivanenko A, Johnson K. Sleep in children with autistic spectrum disorder. Sleep Med 2010;11(7):659-664

84 Sivertsen B, Posserud MB, Gillberg C, Lundervold AJ, Hysing M. Sleep problems in children with autism spectrum problems: a longitudinal population-based study. Autism 2012;16(2): 139-150

85 Hollway JA, Aman MG, Butter E. Correlates and risk markers for sleep disturbance in participants of the Autism Treatment Network. J Autism Dev Disord 2013;43(12):2830-2843

86 Rossignol DA, Frye RE. Melatonin in autism spectrum disorders: a systematic review and meta-analysis. Dev Med Child Neurol 2011;53(9):783-792

87 Miano S, Bruni O, Elia M, et al. Sleep in children with autistic spectrum disorder: a questionnaire and polysomnographic study. Sleep Med 2007;9(1):64-70

88 Johnson KP, Malow BA. Assessment and pharmacologic treatment of sleep disturbance in autism. Child Adolesc Psychiatr Clin N Am 2008;17(4):773-785, viii

89 Bruni O, Ferri R, Vittori E, et al. Sleep architecture and NREM alterations in children and adolescents with Asperger syndrome. Sleep 2007;30(11):1577-1585

90 Malow BA, Marzec ML, McGrew SG, Wang L, Henderson LM, Stone WL. Characterizing sleep in children with autism spectrum disorders: a multidimensional approach. Sleep 2006;29(12): 1563-1571

91 Vriend JL, Corkum PV, Moon EC, Smith IM. Behavioral interventions for sleep problems in children with autism spectrum disorders: current findings and future directions. J Pediatr Psychol 2011;36(9):1017-1029

92 Andersen IM, Kaczmarska J, McGrew SG, Malow BA. Melatonin for insomnia in children with autism spectrum disorders. J Child Neurol 2008;23(5):482-485
93 Malow B, Adkins KW, McGrew SG, et al. Melatonin for sleep in children with autism: a controlled trial examining dose, tolerability, and outcomes. J Autism Dev Disord 2012;42(8): 1729-1737, author reply 1738

94 Wasdell MB, Jan JE, Bomben MM, et al. A randomized, placebocontrolled trial of controlled release melatonin treatment of delayed sleep phase syndrome and impaired sleep maintenance in children with neurodevelopmental disabilities. J Pineal Res 2008;44(1):57-64

95 Bax M, Goldstein M, Rosenbaum P, et al; Executive Committee for the Definition of Cerebral Palsy. Proposed definition and classification of cerebral palsy, April 2005. Dev Med Child Neurol 2005; 47(8):571-576

96 Simard-Tremblay E, Constantin E, Gruber R, Brouillette RT, Shevell M. Sleep in children with cerebral palsy: a review. J Child Neurol 2011;26(10):1303-1310

97 Newman CJ, O'Regan M, Hensey O. Sleep disorders in children with cerebral palsy. Dev Med Child Neurol 2006;48(7):564-568

98 Romeo DM, Brogna C, Musto E, et al. Sleep disturbances in preschool age children with cerebral palsy: a questionnaire study. Sleep Med 2014;15(9):1089-1093

99 Shintani T, Asakura K, Ishi K, Yoshida M, Kataura A, Ogasawara H. Obstructive sleep apnea in children with cerebral palsy. Nippon Jibiinkoka Gakkai Kaiho 1998;101(3):266-271

100 Wiggs L, Stores G. Severe sleep disturbance and daytime challenging behaviour in children with severe learning disabilities. J Intellect Disabil Res 1996;40(Pt 6):518-528

101 Shibagaki M, Kiyono S, Takeuchi T. Nocturnal sleep in mentally retarded infants with cerebral palsy. Electroencephalogr Clin Neurophysiol 1985;61(6):465-471

102 Masters IB, Harvey JM, Wales PD, O’Callaghan MJ, Harris MA. Clinical versus polysomnographic profiles in children with obstructive sleep apnoea. J Paediatr Child Health 1999;35(1):49-54

103 Romeo DM, Brogna C, Quintiliani M, et al. Sleep disorders in children with cerebral palsy: neurodevelopmental and behavioral correlates. Sleep Med 2014;15(2):213-218

104 Hsiao KH, Nixon GM. The effect of treatment of obstructive sleep apnea on quality of life in children with cerebral palsy. Res Dev Disabil 2008;29(2):133-140

105 Kosko JR, Derkay CS. Uvulopalatopharyngoplasty: treatment of obstructive sleep apnea in neurologically impaired pediatric patients. Int J Pediatr Otorhinolaryngol 1995;32(3):241-246

106 Myatt HM, Beckenham EJ. The use of diagnostic sleep nasendoscopy in the management of children with complex upper airway obstruction. Clin Otolaryngol Allied Sci 2000;25(3):200-208

107 Becker DA, Fennell EB, Carney PR. Sleep disturbance in children with epilepsy. Epilepsy Behav 2003;4(6):651-658

108 McCarty SF, Gaebler-Spira D, Harvey RL. Improvement of sleep apnea in a patient with cerebral palsy. Am J Phys Med Rehabil 2001;80(7):540-542

109 Owens JA, Rosen CL, Mindell JA, Kirchner HL. Use of pharmacotherapy for insomnia in child psychiatry practice: A national survey. Sleep Med 2010;11(7):692-700

110 Braam W, van Geijlswijk I, Keijzer H, Smits MG, Didden R, Curfs LM. Loss of response to melatonin treatment is associated with slow melatonin metabolism. J Intellect Disabil Res 2010;54(6):547-555

111 Appleton RE, Gringras P. Melatonin: helping to MEND impaired sleep. Arch Dis Child 2013;98(3):216-217

112 Braam W, Smits MG, Didden R, Korzilius H, Van Geijlswijk IM, Curfs LM. Exogenous melatonin for sleep problems in individuals with intellectual disability: a meta-analysis. Dev Med Child Neurol 2009;51(5):340-349

113 Phillips L, Appleton RE. Systematic review of melatonin treatment in children with neurodevelopmental disabilities and sleep impairment. Dev Med Child Neurol 2004;46(11):771-775

114 Appleton RE, Jones AP, Gamble C, et al. The use of MElatonin in children with neurodevelopmental disorders and impaired 
sleep: a randomised, double-blind, placebo-controlled, parallel study (MENDS). Health Technol Assess 2012;16(40):i-239

115 Lo HS, Yang CM, Lo HG, Lee CY, Ting H, Tzang BS. Treatment effects of gabapentin for primary insomnia. Clin Neuropharmacol 2010; 33(2):84-90

116 Robinson AA, Malow BA. Gabapentin shows promise in treating refractory insomnia in children. J Child Neurol 2013;28(12): 1618-1621

117 Nguyen M, Tharani S, Rahmani M, Shapiro M. A review of the use of clonidine as a sleep aid in the child and adolescent population. Clin Pediatr (Phila) 2014;53(3):211-216

118 Delbarre B, Schmitt H. Sedative effects of alpha-sympathomimetic drugs and their antagonism by adrenergic and cholinergic blocking drugs. Eur J Pharmacol 1971;13(3):356-363

119 Ingrassia A, Turk J. The use of clonidine for severe and intractable sleep problems in children with neurodevelopmental disordersa case series. Eur Child Adolesc Psychiatry 2005;14(1):34-40

120 Ming X, Gordon E, Kang N, Wagner GC. Use of clonidine in children with autism spectrum disorders. Brain Dev 2008;30(7):454-460
121 Hollway JA, Aman MG. Pharmacological treatment of sleep disturbance in developmental disabilities: a review of the literature. Res Dev Disabil 2011;32(3):939-962

122 Spiller HA, Klein-Schwartz W, Colvin JM, Villalobos D, Johnson $\mathrm{PB}$, Anderson DL. Toxic clonidine ingestion in children. J Pediatr 2005;146(2):263-266

123 Arens R, Wright B, Elliott J, et al. Periodic limb movement in sleep in children with Williams syndrome. J Pediatr 1998;133(5): 670-674

124 Goraya JS, Virdi V, Parmar V. Recurrent nocturnal tongue biting in a child with hereditary chin trembling. J Child Neurol 2006; 21(11):985-987

125 Thirumalai SS, Shubin RA, Robinson R. Rapid eye movement sleep behavior disorder in children with autism. J Child Neurol 2002; 17(3):173-178

126 Edinger JD, Fins AI, Sullivan RJ, Marsh GR, Dailey DS, Young M. Comparison of cognitive-behavioral therapy and clonazepam for treating periodic limb movement disorder. Sleep 1996;19(5): $442-444$ 